\title{
Comet Shoemaker-Levy 9: No effect on the Io plasma torus
}

\author{
Michael E. Brown ${ }^{1,3,4}$, Elisabeth J. Moyer ${ }^{2}$, Antonin H. Bouchez ${ }^{3}$, and Hyron \\ Spinrad $^{3}$
}

\begin{abstract}
Observations of the Io plasma torus made before, during, and after the impact of Comet Shoemaker-Levy 9 with Jupiter reveal no cometinduced changes. Three weeks of high spectralresolution ground-based visible spectroscopy show no changes larger than typical day-to-day variations in the torus densities, ion temperatures, or rotation velocities. Comparison with six months of identically obtained data from 1991 and 1992 also shows no differences.
\end{abstract}

\section{Introduction}

A large body crossing the magnetosphere and impacting Jupiter could potentially affect the Io plasma torus in a number of ways. Possibilities might include injection of new material into the torus, heating or cooling of torus electrons and ions by collisions, or changes in the ionospheric current loop which controls torus corotation. In the months before the Comet Shoemaker-Levy 9 impact, several specific predictions were made of effects that cometary material might have on the torus. Predictions included a brightening of the torus due to pick-up heating of cometary materials in the outer Jovian magnetosphere [Herbert, 1994] and a slowing of the torus rotation due to decreased Jovian ionospheric conductivity [Cravens, 1994]. In contrast, others predicted that the comet would have no effect on the torus because the cometary production rate would be small compared to the ongoing mass injection from Io [Dessler and Hill, 1994].

To search for these or other effects, we observed the torus each night for three weeks centered on the impact week and looked for changes. We also compared the data to 6 months of identical observations of the Jupiter/torus system obtained during 1991/2.

\footnotetext{
${ }^{1}$ Lunar and Planetary Laboratory, University of Arizona

${ }^{2}$ Division of Geological and Planetary Sciences, Caltech

${ }^{3}$ Department of Astronomy, University of California at Berkeley

${ }^{4}$ Hubble Fellow
}

Copyright 1995 by the American Geophysical Union.

Paper number 95GL00904

0094-8534/95/95GL-00904\$03.00
This comparison with a long baseline of past observations is particularly important because the torus has many poorly understood short- and long-term variations [see review by Schneider et al., 1989, and examples in Brown, 1994a] that could be mistaken for cometaryinduced changes. From these observations, we find no changes larger than the typical day-to-day variations in the torus density, ion temperature, or velocity structures before, during, or after the impacts.

\section{Observations and Results}

We observed the torus from 8 to 29 July 1994 using the Lick Observatory $0.6-\mathrm{m}$ coudé auxiliary telescope coupled to the Hamilton echelle spectrograph. The observations are high-resolution $(\lambda / \Delta \lambda \sim 40000)$ long slit (slit length $\sim 6$ arcminutes) spectra covering the torus [SII] (i.e., $\mathrm{S}^{+}$) emission doublet at 6717 and $6731 \AA$. For each 40 minute CCD integration the spectral slit was aligned parallel to the Jovian centrifugal equator (the plane of the torus) and centered on Jupiter. Emission from Jupiter was attenuated by covering the center of the slit with a strong neutral density filter. All of the spectra were reduced identically by the methods described in detail in Brown [1994a, 1994b].

Three primary pieces of information - the velocity, temperature, and intensity as a function of distance are extracted from each spectrum. The velocity of the torus is obtained by fitting a gaussian profile to the emission line at each distance; the center of the gaussian profile is assumed to be the velocity of the torus at that spot. The torus very nearly corotates with the $870 \mathrm{deg}$ day $^{-1}$ rotation rate of the Jovian magnetic field, and the high resolution spectra allow us to precisely measure deviations from the corotation velocity. The $\mathrm{S}^{+}$ ion temperature as a function of distance is calculated from the fitted width of the gaussian. The [SII] $6731 \AA$ emission intensity as a function of position along the slit is obtained by integrating all emissions within \pm 40 $\mathrm{km} \mathrm{s}^{-1}$ of the velocity of the emission line and normalizing by the intensity of the Jovian continuum. Figure 1 compares the average measured intensity, velocity, and temperature structure for the 38 spectra from 8 to 15 July (before the impacts) and the 45 spectra from 16 to 29 July (during and after the impacts). Comparisons made with the data grouped in different ways give identical results.

\section{Intensity}

The intensity profile measured in the torus the week before the impacts is similar to the average profile mea- 


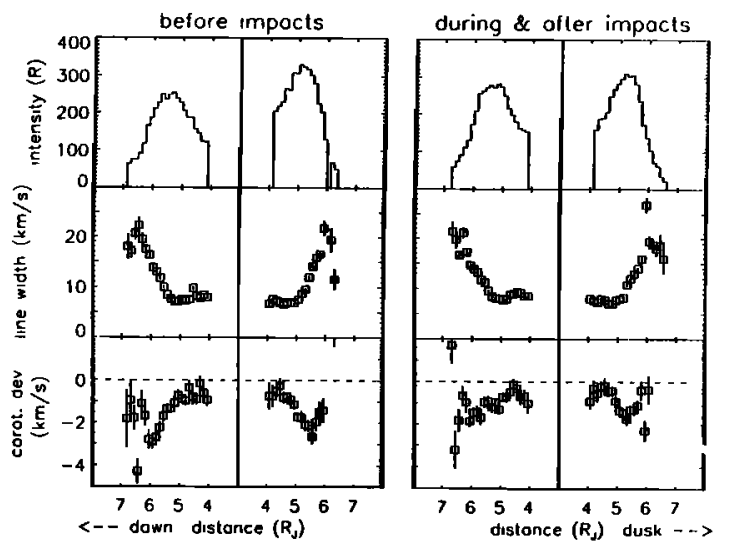

Figure 1. A comparison of the intensities, temperatures, and velocities measured for the [SII] $6731 \AA$ emission of the torus before (9-15 July 1994) and during and after (16-29 July 1994) the SL9 impacts. The parameters are plotted only for the range $4-7.25 R_{J}$ on the dawn and dusk sides of the torus. The intensity is in Rayleighs. The temperature plotted is the width of a single gaussian fit to the emission line. The instrumental profile has a width of $3 \mathrm{~km} \mathrm{~s}^{-1}$. The corotation deviation is the difference between the measured velocity and the corotation velocity at the point. A strictly corotating torus would fall on the dotted lines. All measured velocities are slightly slower than corotation.

sured during an eight month period in 1991 and 1992 [Brown 1994a]. The profile measured the week after the impacts is generally unaffected. The only visible change is a slight difference in the dawn/dusk intensity ratio, but changes of this small magnitude are commonly seen in the 1991/2 data. No effects due to the comet are apparent.

The torus intensity in the [SII] $6731 \AA$ emission is primarily a function of number density. The lack of an observable intensity difference indicates that no change in the density greater than $\sim 3 \%$ occurred during the time of the impacts. In addition, as the spatial structure of the emission intensity in the torus is likely related to the details of ion heating and pickup, we regard the close resemblance between the before and after intensity profiles as evidence that ion pickup was unaffected by the impacts during the time of the observations.

\section{Temperature}

The temperature structure of the torus both before and after the impacts is nearly identical to that measured in 1991/2. Torus core ion temperatures, as measured by fitting a single gaussian to the line profile, are unchanged to within $10 \%$.

However, an injection of energetic particles into the torus might reveal itself by a change not in the core temperature, but by the addition of high-velocity wings to the velocity distribution of the emission lines. Figure 2 shows a comparison between the line profiles measured at $6 \mathrm{RJ}_{\mathrm{J}}$ on the dawn side of the torus before and after the impacts. The two profiles are almost identical even in the high-velocity wings. No observable heating or cooling of torus ions took place due to the impacts.

\section{Velocity}

The pre-impact velocity structure is almost identical to that measured in 1991/2 [Brown 1994b]. The postimpact structure does show slight variations, in particular the velocities between about 5.5 and 6.5 Jovian radii $\left(R_{J}\right)$ are $\sim 1 \mathrm{~km} \mathrm{~s}^{-1}$ higher (closer to corotation) than the same velocities before the impacts. Again, variations of this order of magnitude are typical of the $1991 / 2$ data, so we regard this as insignificant.

The torus rotation is controlled in part by the ionospheric conductivity, which was predicted as a possible change following the impacts [Cravens, 1994]. The deviation from corotation velocity of the torus is inversely proportional to the height-integrated conductivity of the region of the ionosphere that is connected by field-aligned currents to the torus [Hill 1979,1980]. The lack of a detectable slow-down in the torus shows that the height-integrated ionospheric conductivity at the torus L-shell was unaffected by the impacts. Decreases in this Pederson conductivity of as little as a factor of two would have been easily detectable.

\section{Individual spectra}

In addition to searching for average effects before, during, and after the impacts, we examined the individual spectra to search for any short-term changes. A detailed look at these spectra reveals no variations greater than those observed in 1991/2 in any of the measured parameters, as demonstrated by Figure 3, which shows the torus intensities for the individual observations.

\section{Why was nothing seen?}

With the unexpectedly large effects observable on Jupiter as a result of the comet impacts, there was heightened expectation for observable torus changes.
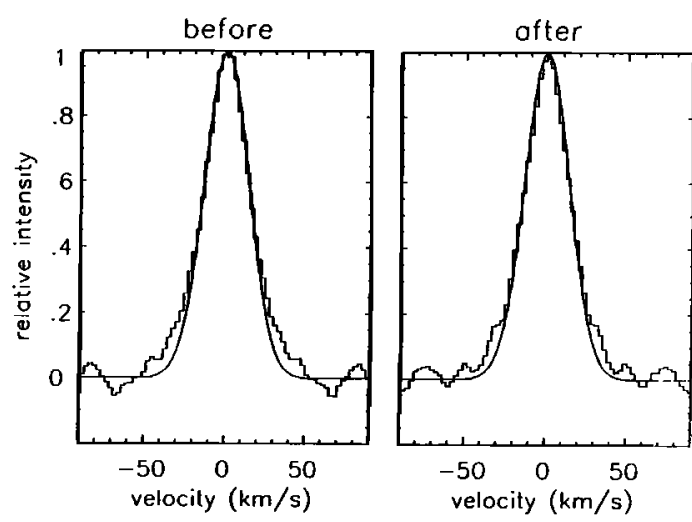

Figure 2. A comparison of average [SII] $6731 \AA$ emission lines at $6 R_{J}$ on the dawn side of the torus before and after the impacts. The solid line in both spectra is a single-gaussian fit to the core of the before-impact line profile. In addition to having identical core temperatures, the high velocity wings of the profiles are also identical. 


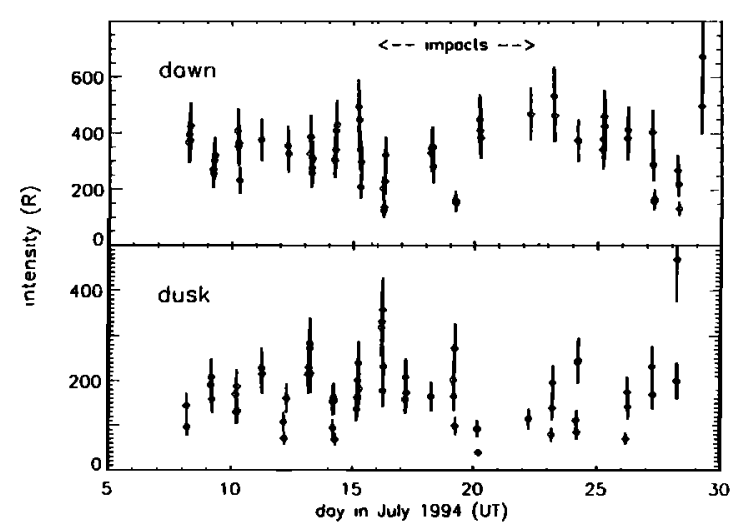

Figure 3. Average emission between 5.8 and $6.2 R_{J}$ on the dawn and dusk sides of the torus for each observation. The impacts occur between UT 16.83 and $22.33 \mathrm{July}$, which are marked by dashed lines. No effects. from the impacts are visible. The general anticorrelation between the dawn and dusk intensities results from rotating bright spots in the torus (see Brown, 1994a).

Yet despite even the large-scale magnetospheric perturbations observable in the Jovian synchrotron emissions [de Pater et al., 1995], the visible torus remained unaltered. We give several potential explanations for the lack of a torus effect.

One always present difficulty with looking for any changes in the torus is that with its many poorly understood variations, a very large and unusual effect would be required before we could definitively attribute it to the fragment impacts. For example, in the past the torus has been seen to brighten by almost a factor of two in under a week with no apparent cause [Brown, 1994a]. Such behavior during the impacts would have been intriguing, but not entirely convincing. Because of its large variations, the standard of proof for an induced torus change must remain high.

These observations were originally designed (in 1991) to detect very small changes in the torus rotation velocity, so the detection of impact-induced rotation changes was one of our primary observational goals. Unfortunately, torus slowdowns due to reduced ionospheric conductivity might not have occurred even if the ionosphere was affected by the impact. Magnetospheric corotation is controlled by the height-integrated Pederson conductivity. This conductivity is thought to be dominated by the metallic ion layers [Atreya, 1989] which should be unaffected by the water chemistry predicted by Cravens [1994]. In addition, the torus is affected only by the conductivity at the foot of the torus L-shell; the time for ionospheric chemical perturbations to propagate to the polar regions from the impact sites is unknown.

The simplest overall explanation for the lack of any other observable torus effect is probably that given by Dessler and Hill [1994]: the fragments were too small and in the wrong place. The production rate was insignificant compared to the large amount of mass con- tinuously ejected from Io, and the southern approaching trajectory was unfavorable for equatorial torus interaction. Thus there was no change in torus densities, ion temperatures, or rotation velocities larger than the normal day-to-day variations.

Acknowledgments. These observations were made possible by NSF Grant \# FD-93-22119. Additional support for this work was provided by NASA through grant number HF-1056.01-94A from the Space Telescope Science Institute, which is operated by the Association of Universities for Research in Astronomy, Inc., under NASA contract NAS526555. MEB and EJM acknowledge the support of NASA and NSF graduate fellowships, respectively, and thank Jim Burroughs for making the long stay at Lick Observatory bearable.

\section{References}

Atreya, S.K., Atmosphere and ionospheres of the outer planets and their satellites, in Physics and Chemsitry of Space, 15, Springer-Verlag, Berlin, 1986.

Brown, M.E., The structure and variability of the Io plasma torus, Ph.D. dissertation, University of California at Berkeley, 1994b.

Brown, M.E., Observations of mass loading in the Io plasma torus, Geophys. Res. Lett., 21, 847-850, 1994a.

Cravens, T.E., Comet Shoemaker-Levy-9 Impact With Jupiter: Aeronomical Predictions, Geophys. Res. Lett., 21, 1075, 1994.

de Pater, I., et al., The outburst of Jupiter's synchrotron radiation following the impact of Comet $P$ /ShoemakerLevy 9, submitted to Science, 1995.

Dessler, A.J., and T.W. Hill, Some interactions between dust from Comet Shoemaker-Levy 9 and Jupiter, Geophys. Res. Lett., 21, 1043, 1994.

Herbert, F., The impact of Comet Shoemaker-Levy 9 on the Jovian magnetosphere, Geophys. Res. Lett., 21, 1047, 1994.

Hill, T.W., Inertial limit on corotation, J. Geophys. Res., $84,6554,1979$.

Hill, T.W., Corotation lag in Jupiters magnetosphere: A comparison of observation and theory, Science, 207, 301, 1980.

Schneider, N.M., M.A. McGrath, and W.H. Smyth, Io's atmosphere and neutral clouds, in Time-Variable Phenomena in the Jovian System, Belton, M.J.S, R.A. West, and J. Rahe, eds., NASA SP-494, 1989.

Strobel, D.F., Energetics, luminosity, and spectroscopy of Io's torus, Time Variable Phenomena in the Jovian System, edited by M.J.S. Belton, R.A. West, and J. Rahe, eds., NASA SP-494, 1989.

M. E. Brown, Lunar and Planetary Laboratory, University of Arizona, Tucson, AZ 85721 (email: mbrown@lpl.arizona.edu)

A. H. Bouchez and H. Spinrad, Astronomy Department, University of California, Berkeley, CA 94720.

E. J. Moyer, Division of Geological and Planetary Sciences, Caltech, Pasadena, CA 91125.

(received August 15, 1994; revised February 6, 1995; accepted February 13, 1995.) 